\title{
7 Arithmetic operations on generalized semielliptic intuitionistic fuzzy numbers and their application in multicriteria decision making
}

\begin{abstract}
This chapter attempts to initiate a novel generalized semielliptic intuitionistic fuzzy number (GSEIFN) along with basic arithmetic operations on GSEIFNs. Furthermore, an advanced ranking method for GSEIFN is proposed. In the end, a multicriteria decision-making problem has been carried out by using the proposed GSEIFN and the ranking approach to exhibit the legality and applicability.
\end{abstract}

\subsection{Introduction}

Multicriteria decision making (MCDM) is a common activity in our daily life, such as choosing a car, selecting a candidate in an interview for the post of a teacher, and choosing or buying the best book or the other things from online shopping apps. It is an action or a procedure of choosing the best option from the accessible options through multiple criteria. In making decision, due to the presence of conflicting criteria or vague information, it is impossible to make proper decision by using a traditional set theory. To deal with such uncertain or vague data, Zadeh [1] introduced the fuzzy set theory (FST), which deals with those elements that partially belonged to the set. Later, Atanassov [2] extended FST, which is known as intuitionistic fuzzy set (IFS), which deals with both elements that are partially belonged to the set and not belonged to the set. IFS deals with such situations in an enhanced manner in comparison to FST. Due to growing complexities in making decisions, several intuitionistic fuzzy numbers (IFNs) have been developed [3-5]. More often, for simplicity, triangular or trapezoidal IFNs (TrIFNs) are employed to solve decision-making problems. However, in some realworld situations, the interactions between available system factors are found to be more complicated and in such situations triangular or trapezoidal types of IFNs fail to provide proper result. To make a proper decision, it is more essential to reconstruct an appropriate IFN. To overcome such difficulties, generalized semielliptic (GSE) type of IFN may be developed by making more suitable to handle such complex environments.

In MCDM problems, ranking of IFNs plays an efficient role. In literature, a few numbers of ranking approaches are encountered based on value and ambiguity [6], centroid point [7], centroid [3], magnitude [8], area [9], score and accuracy function

Palash Dutta, Bornali Saikia, Department of Mathematics, Dibrugarh University, Dibrugarh, India 
[10], possibility mean [11], mean value [12], and improved possibility degree [13]. The approaches $[3,4,6,14-24]$ focused on the value as well as the ambiguity-based ranking method of IFNs and applied in MCDM problems. The approaches in [7] and [3] dealt with the centroid-based ranking method, the approach in [21] presented a ranking technique for IFNs by using the distance of each IFN from the fuzzy origin, and the approach in [9] developed a ranking method based on $(\alpha, \beta)$-cuts and area of IFNs. Notwithstanding having some primacy of ranking approaches, these approaches have some deficiencies and do not provide a proper result, which produces predicament for judgment architects in any decision-making problem. To overcome such limitations, a new ranking approach has been developed based on the consummated exponential mean. Furthermore, a comparative analysis of the present ranking approach and other available techniques has been presented to exhibit the validity of the same. In this chapter, an effort has been made to introduce generalized semielliptic IFN (GSEIFN) and perform its fundamental operations of GSEIFNs. Finally, an MCDM problem is being solved to demonstrate the existence and significance of proposed IFNs and proposed ranking approach.

\subsection{Preliminaries}

Definition 7.1 ([2]). An IFS $B$ defined on the universal set $X$ is denoted by $B=\left\{\left(x, \mu_{B}(x), v_{B}(x)\right), x \in X\right\} . \mu_{B}: X \rightarrow[0,1]$ and $v_{B}: X \rightarrow[0,1]$ are respective membership function (MF) and nonmembership function (NMF) such that $0 \leq \mu_{B}(x)+v_{B}(x) \leq 1$. The amount $\pi_{B}(x)=1-\mu_{B}(x)-v_{B}(x)$ is called the degree of hesitancy.

Definition 7.2 ([6]). An $\alpha$-cut of $B$ is defined as

$$
{ }^{\alpha} B=\left\{x \mid \mu_{B}(x) \geq \alpha\right\}
$$

For IFN $\alpha$-cut produces a closed interval, say ${ }^{\alpha} B=\left[L_{B}(\alpha), R_{B}(\alpha)\right]$.

Definition 7.3 ([6]). An $\beta$-cut of $B$ is defined as

$$
{ }^{\beta} B=\left\{x \mid v_{B}(x) \leq \beta\right\}
$$

For an IFN the $\alpha$-cut produces a closed interval, say ${ }^{\beta} B=\left[L_{B}(\beta), R_{B}(\beta)\right]$.

Definition $7.4([6]) . \quad$ An $(\alpha, \beta)$-cut of $B$ is defined as

$$
{ }^{(\alpha, \beta)} B=\left\{x \mid \mu_{B}(x) \geq \alpha, v_{B}(x) \leq \beta\right\}
$$

such that $0 \leq \alpha \leq 1,0 \leq \beta \leq 1$, and $0 \leq \alpha+\beta \leq 1$. 
Definition 7.5 ([18]). The values of IFN $B$ of MF and NMF are denoted by $V_{\mu}(B)$ and $V_{v}(B)$, respectively, as

$$
\begin{gathered}
V_{\mu}(B)=\int_{0}^{w}\left[L_{B}(\alpha)+R_{B}(\alpha)\right] \alpha d \alpha \\
V_{v}(B)=\int_{\eta}^{1}\left[L_{B}(\beta)+R_{B}(\beta)\right](1-\beta) d \beta
\end{gathered}
$$

Definition 7.6 ([18]). The ambiguities of IFN $B$ of MF and NMF are denoted by $A_{\mu}(B)$ and $A_{v}(B)$, respectively, as

$$
\begin{gathered}
A_{\mu}(B)=\int_{0}^{w}\left[L_{B}(\alpha)-R_{B}(\alpha)\right] \alpha d \alpha \\
A_{\nu}(B)=\int_{\eta}^{1}\left[L_{B}(\beta)-R_{B}(\beta)\right](1-\beta) d \beta
\end{gathered}
$$

Definition 7.7. The mean of IFN $B$ are denoted $M_{\mu}(B)$ and $M_{v}(B)$, respectively, as

$$
\begin{aligned}
& M_{\mu}(B)=\frac{1}{2} \int_{0}^{w}\left[L_{B}(\alpha)+R_{B}(\alpha)\right] d \alpha \\
& M_{\nu}(B)=\frac{1}{2} \int_{\eta}^{1}\left[L_{B}(\beta)+R_{B}(\beta)\right] d \beta
\end{aligned}
$$

Definition $7.8([5])$. The general expression of an ellipse centered at $(p, q)$ is

$$
\frac{(x-p)^{2}}{h^{2}}+\frac{(y-q)^{2}}{k^{2}}=1
$$

Assuming that $q=0$ and $k=1$ to construct a normal semielliptic IFN (NSEIFN) $P$, we have

$$
\frac{(x-p)^{2}}{h^{2}}+y^{2}=1
$$

Then the needed MF is

$$
\mu_{P}(x)=\sqrt{1-\frac{(x-p)^{2}}{h^{2}}}, p-h \leq x \leq p+h
$$

In the same way, the NMF can also be constructed as

$$
v_{P}(x)=1-\sqrt{1-\frac{(x-p)^{2}}{h_{1}^{2}}}, p-h_{1} \leq x \leq p+h_{1}
$$


where $h \leq h_{1}$ and $p$ is the core of the NSEIFN $P$, while $h$ and $h_{1}$ indicate support/domain MF and NMF, respectively.

Definition 7.9. The GSEIFN $P=S_{E}\left(p, h_{1}, h_{2} ; w_{1}, \eta_{1}\right)$ on universal set of discourse $X$ is defined by the MF and NMF, respectively, as

$$
\begin{gathered}
\mu_{P}(x)=w_{1} \sqrt{1-\frac{(x-p)^{2}}{h_{1}^{2}}}, p-h_{1} \leq x \leq p+h_{1} \\
v_{P}(x)=1-\left(1-\eta_{1}\right) \sqrt{1-\frac{(x-p)^{2}}{h_{2}^{2}}}, p-h_{2} \leq x \leq p+h_{2}
\end{gathered}
$$

Here $h_{1} \leq h_{2}$.

Definition 7.10. Consider $P=S_{E}\left(p, h_{1}, h_{2} ; w_{1}, \eta_{1}\right)$ be a GSEIFN. Then $(\alpha, \beta)$-cut of the GSEIFN is

$$
\begin{aligned}
& { }^{\alpha} P=\left[p-h_{1} \sqrt{1-\left\{\frac{\alpha}{w_{1}}\right\}^{2}}, p+h_{1} \sqrt{1-\left\{\frac{\alpha}{w_{1}}\right\}^{2}}\right] \\
& { }^{\beta} P=\left[p-h_{2} \sqrt{1-\left\{\frac{1-\beta}{1-\eta_{1}}\right\}^{2}}, p+h_{2} \sqrt{1-\left\{\frac{1-\beta}{1-\eta_{1}}\right\}^{2}}\right]
\end{aligned}
$$

\subsection{Elementary operations on GSEIFNs}

Here, we use $(\alpha, \beta)$-cut method to perform some arithmetic operations of GSEIFNs and also discuss some numerical examples. Suppose that $P=S_{E}\left(p, h_{1}, h_{2} ; w_{1}, \eta_{1}\right)$ and $Q=S_{E}\left(q, k_{1}, k_{2} ; w_{2}, \eta_{2}\right)$ are two GSEIFNs whose MF and NMFs are defined as follows:

$$
\begin{gathered}
\mu_{P}(x)=w_{1} \sqrt{1-\frac{(x-p)^{2}}{h_{1}^{2}}}, p-h_{1} \leq x \leq p+h_{1} \\
v_{P}(x)=1-\left(1-\eta_{1}\right) \sqrt{1-\frac{(x-p)^{2}}{h_{2}^{2}}}, p-h_{2} \leq x \leq p+h_{2}
\end{gathered}
$$

Here $h_{1} \leq h_{2}$

$$
\mu_{Q}(x)=w_{2} \sqrt{1-\frac{(x-q)^{2}}{k_{1}^{2}}}, q-k_{1} \leq x \leq q+k_{1}
$$




$$
v_{Q}(x)=1-\left(1-\eta_{2}\right) \sqrt{1-\frac{(x-q)^{2}}{k_{2}^{2}}}, q-k_{2} \leq x \leq q+k_{2}
$$

Here $k_{1} \leq k_{2}$.

Now we find out that the $(\alpha, \beta)$-cuts of $P$ and $Q$ are

$$
\begin{aligned}
& { }^{\alpha} P=\left[p-h_{1} \sqrt{1-\left\{\frac{\alpha}{w_{1}}\right\}^{2}}, p+h_{1} \sqrt{1-\left\{\frac{\alpha}{w_{1}}\right\}^{2}}\right] \\
& { }^{\beta} P=\left[p-h_{2} \sqrt{1-\left\{\frac{1-\beta}{1-\eta_{1}}\right\}^{2}}, p+h_{2} \sqrt{1-\left\{\frac{1-\beta}{1-\eta_{1}}\right\}^{2}}\right] \\
& { }^{\alpha} Q=\left[q-k_{1} \sqrt{1-\left\{\frac{\alpha}{w_{2}}\right\}^{2}}, q+k_{1} \sqrt{1-\left\{\frac{\alpha}{w_{2}}\right\}^{2}}\right] \\
& { }^{\beta} Q=\left[q-k_{2} \sqrt{1-\left\{\frac{1-\beta}{1-\eta_{2}}\right\}^{2}}, q+k_{2} \sqrt{1-\left\{\frac{1-\beta}{1-\eta_{2}}\right\}^{2}}\right]
\end{aligned}
$$

Here we also use $w_{1}=\min \left(w_{1}, w_{2}\right)$ and $\eta_{1}=\max \left(\eta_{1}, \eta_{2}\right)$ to perform arithmetic operations.

\subsubsection{Addition of GSEIFNs}

For the evaluation of sum of GSEIFNs $P$ and $Q$, it is required to sum the $\alpha$-cuts and $\beta$-cuts of $P$ and $Q$ via interval operations and here we assume $w_{1}=\min \left(w_{1}, w_{2}\right)$, $\eta_{1}=\max \left(\eta_{1}, \eta_{2}\right)$ :

$$
\begin{gathered}
{ }^{\alpha} P+{ }^{\alpha} Q=\left[(p+q)-\left(h_{1}+k_{1}\right) \sqrt{1-\left\{\frac{\alpha}{w_{1}}\right\}^{2}},(p+q)+\left(h_{1}+k_{1}\right) \sqrt{1-\left\{\frac{\alpha}{w_{1}}\right\}^{2}}\right] \\
{ }^{\beta} P+{ }^{\beta} Q=\left[(p+q)-\left(h_{2}+k_{2}\right) \sqrt{1-\left\{\frac{1-\beta}{1-\eta_{1}}\right\}^{2}},(p+q)+\left(h_{2}+k_{2}\right) \sqrt{1-\left\{\frac{1-\beta}{1-\eta_{1}}\right\}^{2}}\right]
\end{gathered}
$$

Now for getting proper MF $\mu_{P+Q}(x)$ and NMF $\nu_{P+Q}(x)$, it is important to equate initial component and final component of eqs. (7.1) and (7.2) with $x$ which give

$$
x=(p+q)-\left(h_{1}+k_{1}\right) \sqrt{1-\left\{\frac{\alpha}{w_{1}}\right\}^{2}}, x=(p+q)-\left(h_{2}+k_{2}\right) \sqrt{1-\left\{\frac{1-\beta}{1-\eta_{1}}\right\}^{2}}
$$




$$
x=(p+q)+\left(h_{1}+k_{1}\right) \sqrt{1-\left\{\frac{\alpha}{w_{1}}\right\}^{2}}, x=(p+q)+\left(h_{2}+k_{2}\right) \sqrt{1-\left\{\frac{1-\beta}{1-\eta_{1}}\right\}^{2}}
$$

Next, it is needed to express $\alpha$ in terms of $x$ by taking $\alpha \geq 0, \alpha \leq w_{1}$ and $\alpha \leq w_{1}, \alpha \geq 0$ in eq. (7.1) we have

$$
\alpha=w_{1} \sqrt{1-\left\{\frac{x-(p+q)}{h_{1}+k_{1}}\right\}^{2}}, \alpha=w_{1} \sqrt{1-\left\{\frac{x-(p+q)}{h_{1}+k_{1}}\right\}^{2}}
$$

We get the domain of $x$,

$$
x \in\left[\left(p-h_{1}\right)+\left(q-k_{1}\right),(p+q)\right], x \in\left[(p+q),\left(p+h_{1}\right)+\left(q+k_{1}\right)\right]
$$

In the same way, $\beta$ can be expressed in terms of $x$ by taking $\beta \leq \eta_{1}, \beta \geq 0$ and $\beta \geq 0$, $\beta \leq \eta_{1}$ in eq. (7.2) we have

$$
\beta=1-\left(1-\eta_{1}\right) \sqrt{1-\left\{\frac{x-(p+q)}{h_{2}+k_{2}}\right\}^{2}}, \beta=1-\left(1-\eta_{1}\right) \sqrt{1-\left\{\frac{x-(p+q)}{h_{2}+k_{2}}\right\}^{2}}
$$

We get the domain of $x$,

$$
x \in\left[\left(p-h_{2}\right)+\left(q-k_{2}\right),(p+q)\right], x \in\left[(p+q),\left(p+h_{2}\right)+\left(q+k_{2}\right)\right]
$$

Thus, the MF $\mu_{P+Q}(x)$ and NMF $v_{P+Q}(x)$ of $P+Q$ are

$$
\begin{gathered}
\mu_{(P+Q)}=w_{1} \sqrt{1-\left\{\frac{x-(p+q)}{h_{1}+k_{1}}\right\}^{2}}, x \in\left[\left(p-h_{1}\right)+\left(q-k_{1}\right),\left(p+h_{1}\right)+\left(q+k_{1}\right)\right] \\
v_{(P+Q)}=1-\left(1-\eta_{1}\right) \sqrt{1-\left\{\frac{x-(p+q)}{h_{2}+k_{2}}\right\}^{2}}, x \in\left[\left(p-h_{2}\right)+\left(q-k_{2}\right),\left(p+h_{2}\right)+\left(q+k_{2}\right)\right]
\end{gathered}
$$

\subsubsection{Subtraction of GSEIFNs}

For evaluation of subtraction of GSEIFNs $P$ and $Q$, here, it is also required to subtract the corresponding $\alpha$-cuts and $\beta$-cuts of $P$ and $Q$ via interval operations and considering $w_{1}=\min \left(w_{1}, w_{2}\right), \eta_{1}=\max \left(\eta_{1}, \eta_{2}\right)$, we have

$$
\begin{gathered}
{ }^{\alpha} P-{ }^{\alpha} Q=\left[(p-q)-\left(h_{1}+k_{1}\right) \sqrt{1-\left\{\frac{\alpha}{w_{1}}\right\}^{2}},(p-q)+\left(h_{1}+k_{1}\right) \sqrt{1-\left\{\frac{\alpha}{w_{1}}\right\}^{2}}\right] \\
{ }^{\beta} P-{ }^{\beta} Q=\left[(p-q)-\left(h_{2}+k_{2}\right) \sqrt{1-\left\{\frac{1-\beta}{1-\eta_{1}}\right\}^{2}},(p-q)+\left(h_{2}+k_{2}\right) \sqrt{1-\left\{\frac{1-\beta}{1-\eta_{1}}\right\}^{2}}\right]
\end{gathered}
$$


Now, for getting proper MF $\mu_{P-Q}(x)$ and NMF $v_{P-Q}(x)$ of $P-Q$, it is essential to equate both initial and final components with $x$ eqs. (7.3) and (7.4) which give

$$
\begin{aligned}
& x=(p-q)-\left(h_{1}+k_{1}\right) \sqrt{1-\left\{\frac{\alpha}{w_{1}}\right\}^{2}}, x=(p-q)-\left(h_{2}+k_{2}\right) \sqrt{1-\left\{\frac{1-\beta}{1-\eta_{1}}\right\}^{2}} \\
& x=(p-q)+\left(h_{1}+k_{1}\right) \sqrt{1-\left\{\frac{\alpha}{w_{1}}\right\}^{2}}, x=(p-q)+\left(h_{2}+k_{2}\right) \sqrt{1-\left\{\frac{1-\beta}{1-\eta_{1}}\right\}^{2}}
\end{aligned}
$$

Next, it is further essential to express $\alpha$ in terms of $x$ by considering $\alpha \geq 0, \alpha \leq w_{1}$ and $\alpha \leq w_{1}, \alpha \geq 0$ in eq. (7.3) we have

$$
\alpha=w_{1} \sqrt{1-\left\{\frac{x-(p-q)}{h_{1}+k_{1}}\right\}^{2}}, \alpha=w_{1} \sqrt{1-\left\{\frac{x-(p-q)}{h_{1}+k_{1}}\right\}^{2}}
$$

We get the domain of $x$,

$$
x \in\left[\left(p-h_{1}\right)-\left(q+k_{1}\right),(p-q)\right], x \in\left[(p-q),\left(p+h_{1}\right)-\left(q-k_{1}\right)\right]
$$

In the same fashion, $\beta$ can be expressed in terms of $x$ by taking $\beta \leq \eta_{1}, \beta \geq 0$ and $\beta \geq 0, \beta \leq \eta_{1}$ in eq. (7.4) we have

$$
\beta=1-\left(1-\eta_{1}\right) \sqrt{1-\left\{\frac{x-(p-q)}{h_{2}+k_{2}}\right\}^{2}}, \beta=1-\left(1-\eta_{1}\right) \sqrt{1-\left\{\frac{x-(p-q)}{h_{2}+k_{2}}\right\}^{2}}
$$

We get the domain of $x$,

$$
x \in\left[\left(p-h_{2}\right)-\left(q+k_{2}\right),(p-q)\right], x \in\left[(p-q),\left(p+h_{2}\right)-\left(q-k_{2}\right)\right]
$$

Thus, the MF $\mu_{P-Q}(x)$ and NMF $\nu_{P-Q}(x)$ of $P-Q$ are

$$
\begin{gathered}
\mu_{(P-Q)}=w_{1} \sqrt{1-\left\{\frac{x-(p-q)}{h_{1}+k_{1}}\right\}^{2}}, x \in\left[\left(p-h_{1}\right)-\left(q+k_{1}\right),\left(p+h_{1}\right)-\left(q-k_{1}\right)\right] \\
v_{(P-Q)}=1-\left(1-\eta_{1}\right) \sqrt{1-\left\{\frac{x-(p-q)}{h_{2}+k_{2}}\right\}^{2}}, x \in\left[\left(p-h_{2}\right)-\left(q+k_{2}\right),\left(p+h_{2}\right)-\left(q-k_{2}\right)\right]
\end{gathered}
$$

\subsubsection{Product of GSEIFNs}

For evaluating the product of GSEIFNs $P$ and $Q$, it is required to product the $\alpha$-cuts and $\beta$-cuts of $P$ and $Q$ via interval operations: 


$$
\begin{aligned}
{ }^{\alpha} P^{\alpha} Q= & {\left[\left(p-h_{1} \sqrt{1-\left\{\frac{\alpha}{w_{1}}\right\}^{2}}\right)\left(q-k_{1} \sqrt{1-\left\{\frac{\alpha}{w_{2}}\right\}^{2}}\right),\right.} \\
& \left.\left(p+h_{1} \sqrt{1-\left\{\frac{\alpha}{w_{1}}\right\}^{2}}\right)\left(q+k_{1} \sqrt{1-\left\{\frac{\alpha}{w_{2}}\right\}^{2}}\right)\right] \\
{ }^{\beta} P^{\beta} Q= & {\left[\left(p-h_{2} \sqrt{1-\left\{\frac{1-\beta}{1-\eta_{1}}\right\}^{2}}\right)\left(q-k_{1} \sqrt{1-\left\{\frac{1-\beta}{1-\eta_{2}}\right\}^{2}}\right),\right.} \\
& \left.\left(p+h_{1} \sqrt{1-\left\{\frac{1-\beta}{1-\eta_{1}}\right\}^{2}}\right)\left(q+k_{1} \sqrt{1-\left\{\frac{1-\beta}{1-\eta_{2}}\right\}^{2}}\right)\right]
\end{aligned}
$$

To find $\mathrm{MF} \mu_{P Q}(x)$ and NMF $v_{P Q}(x)$, here too it is needed to equate first and last components to $x$ in eqs. (7.5) and (7.6), which gives

$$
\begin{aligned}
& x=\left(p-h_{1} \sqrt{1-\left\{\frac{\alpha}{w_{1}}\right\}^{2}}\right)\left(q-k_{1} \sqrt{1-\left\{\frac{\alpha}{w_{2}}\right\}^{2}}\right) \\
& x=\left(p+h_{1} \sqrt{1-\left\{\frac{\alpha}{w_{1}}\right\}^{2}}\right)\left(q+k_{1} \sqrt{1-\left\{\frac{\alpha}{w_{2}}\right\}^{2}}\right) \\
& x=\left(p-h_{2} \sqrt{1-\left\{\frac{1-\beta}{1-\eta_{1}}\right\}^{2}}\right)\left(q-k_{1} \sqrt{1-\left\{\frac{1-\beta}{1-\eta_{2}}\right\}^{2}}\right) \\
& x=\left(p+h_{1} \sqrt{1-\left\{\frac{1-\beta}{1-\eta_{1}}\right\}^{2}}\right)\left(q+k_{1} \sqrt{1-\left\{\frac{1-\beta}{1-\eta_{2}}\right\}^{2}}\right)
\end{aligned}
$$

Putting $w_{1}=\min \left(w_{1}, w_{2}\right)$ and expressing $\alpha$ in terms of $x$

$$
\begin{aligned}
& h_{1} k_{1}\left(1-\left\{\frac{\alpha}{w_{1}}\right\}^{2}\right)-\left(p k_{1}+q h_{1}\right)\left(\sqrt{1-\left\{\frac{\alpha}{w_{1}}\right\}^{2}}\right)+p q-x=0 \\
& h_{1} k_{1}\left(1-\left\{\frac{\alpha}{w_{1}}\right\}^{2}\right)+\left(p k_{1}+q h_{1}\right)\left(\sqrt{1-\left\{\frac{\alpha}{w_{1}}\right\}^{2}}\right)+p q-x=0
\end{aligned}
$$

Next, solving the expression by taking $1-\left\{\frac{\alpha}{w_{1}}\right\}^{2}=y^{2}$, we have, 


$$
\begin{gathered}
y=\frac{-\left\{-\left(p k_{1}+q h_{1}\right)\right\} \pm \sqrt{\left\{-\left(p k_{1}+q h_{1}\right)\right\}^{2}-4 h_{1} k_{1}(p q-x)}}{2 h_{1} k_{1}} \\
\alpha=w_{1} \sqrt{1-\left\{\frac{\left(p k_{1}+q h_{1}\right)-\sqrt{\left(p k_{1}+q h_{1}\right)^{2}-4 h_{1} k_{1}(p q-x)}}{2 h_{1} k_{1}}\right\}^{2}}
\end{gathered}
$$

Similarly,

$$
\alpha=w_{1} \sqrt{1-\left\{\frac{\left(p k_{1}+q h_{1}\right)-\sqrt{\left(p k_{1}+q h_{1}\right)^{2}-4 h_{1} k_{1}(p q-x)}}{2 h_{1} k_{1}}\right\}^{2}}
$$

Setting $\alpha \geq 0, \alpha \leq w_{1}$ and $\alpha \leq w_{1}, \alpha \geq 0$ in eq. (7.5), we have

$$
x \in\left[\left(p-h_{1}\right)\left(q-k_{1}\right), p q\right], x \in\left[p q,\left(p+h_{1}\right)\left(q+k_{1}\right)\right]
$$

Putting $\eta_{1}=\max \left(\eta_{1}, \eta_{2}\right)$ and expressing $\beta$ in terms of $x$

$$
\begin{aligned}
& h_{2} k_{2}\left\{1-\left\{\frac{1-\beta}{1-\eta_{1}}\right\}^{2}\right\}-\left(p k_{2}+q h_{2}\right) \sqrt{1-\left\{\frac{1-\beta}{1-\eta_{1}}\right\}^{2}}+p q-x=0 \\
& h_{2} k_{2}\left\{1-\left\{\frac{1-\beta}{1-\eta_{1}}\right\}^{2}\right\}+\left(p k_{2}+q h_{2}\right) \sqrt{1-\left\{\frac{1-\beta}{1-\eta_{1}}\right\}^{2}}+p q-x=0
\end{aligned}
$$

Again solving the expression by taking $1-\left\{\frac{1-\beta}{1-\eta_{1}}\right\}^{2}=q^{2}$, we have

$$
\begin{gathered}
q=\frac{-\left\{-\left(p k_{2}+q h_{2}\right)\right\} \pm \sqrt{\left\{-\left(p k_{2}+q h_{2}\right)\right\}^{2}-4 h_{2} k_{2}(p q-x)}}{2 h_{2} k_{2}} \\
\beta=1-\left(1-\eta_{1}\right) \sqrt{1-\left\{\frac{\left(p k_{2}+q h_{2}\right)-\sqrt{\left(p k_{2}+q h_{2}\right)^{2}-4 h_{2} k_{2}(p q-x)}}{2 h_{2} k_{2}}\right\}^{2}}
\end{gathered}
$$

Similarly

$$
\left.\beta=1-\left(1-\eta_{1}\right) \sqrt{1-\left\{\frac{\left(p k_{2}+q h_{2}\right)-\sqrt{\left(p k_{2}+q h_{2}\right)^{2}-4 h_{2} k_{2}(p q-x)}}{2 h_{2} k_{2}}\right.}\right\}^{2}
$$

Setting $\beta \leq \eta_{1}, \beta \geq 0$ and $\beta \geq 0, \beta \leq \eta_{1}$ in eq. (7.6) we have

$$
x \in\left[\left(p-h_{2}\right)\left(q-k_{2}\right), p q\right], x \in\left[p q,\left(p+h_{2}\right)\left(q+k_{2}\right)\right]
$$

Thus, the MF $\mu_{P Q}(x)$ and NMF $v_{P Q}(x)$ of $P Q$ are 


$$
\begin{gathered}
\mu_{P Q}(x)=w_{1} \sqrt{1-\left\{\frac{\left(p k_{1}+q h_{1}\right)-\sqrt{\left(p k_{1}+q h_{1}\right)^{2}-4 h_{1} k_{1}(p q-x)}}{2 h_{1} k_{1}}\right\}^{2}} \\
\left(p-h_{1}\right)\left(q-k_{1}\right) \leq x \leq\left(p+h_{1}\right)\left(q+k_{1}\right) \\
v_{P Q}(x)=1-\left(1-\eta_{1}\right) \sqrt{1-\left\{\frac{\left(p k_{2}+q h_{2}\right)-\sqrt{\left(p k_{2}+q h_{2}\right)^{2}-4 h_{2} k_{2}(p q-x)}}{2 h_{2} k_{2}}\right\}^{2}} \\
\left(p-h_{2}\right)\left(q-k_{2}\right) \leq x \leq\left(p+h_{2}\right)\left(q+k_{2}\right)
\end{gathered}
$$

\subsubsection{Division of GSEIFNs}

For evaluation division of GSEIFNs $P$ by $Q$, it is essential to divide the $\alpha$-cuts by $\beta$ cuts of $P$ and $Q$ via interval operations:

$$
\begin{gathered}
\frac{{ }^{\alpha} P}{{ }^{\alpha} Q}=\left[\frac{p-h_{1} \sqrt{1-\left\{\frac{\alpha}{w_{1}}\right\}^{2}}}{q+k_{1} \sqrt{1-\left\{\frac{\alpha}{w_{2}}\right\}^{2}}}, \frac{p+h_{1} \sqrt{1-\left\{\frac{\alpha}{w_{1}}\right\}^{2}}}{q-k_{1} \sqrt{1-\left\{\frac{\alpha}{w_{2}}\right\}^{2}}}\right] \\
\frac{{ }^{\beta} P}{\beta Q}=\left[\frac{p-h_{2} \sqrt{1-\left\{\frac{1-\beta}{1-\eta_{1}}\right\}^{2}}}{q+k_{2} \sqrt{\left[1-\left\{\frac{1-\beta}{1-\eta_{2}}\right\}^{2}\right.}}, \frac{p+h_{2} \sqrt{1-\left\{\frac{1-\beta}{1-\eta_{1}}\right\}^{2}}}{q-k_{2} \sqrt{1-\left\{\frac{1-\beta}{1-\eta_{2}}\right\}^{2}}}\right]
\end{gathered}
$$

Now, for getting exact MF $\mu_{\frac{A}{B}}(x)$ and NMF $v_{\frac{A}{B}}(x)$ of $P / Q$, it is required to equate first and last components to $x$ in eqs. (7.7) and (7.8), we have

$$
\begin{aligned}
& x=\frac{p-h_{1} \sqrt{1-\left\{\frac{\alpha}{w_{1}}\right\}^{2}}}{q+k_{1} \sqrt{1-\left\{\frac{\alpha}{w_{2}}\right\}^{2}}}, x=\frac{p+h_{1} \sqrt{1-\left\{\frac{\alpha}{w_{1}}\right\}^{2}}}{q-k_{1} \sqrt{1-\left\{\frac{\alpha}{w_{2}}\right\}^{2}}} \\
& x=\frac{p-h_{2} \sqrt{1-\left\{\frac{1-\beta}{1-\eta_{1}}\right\}^{2}}}{q+k_{2} \sqrt{1-\left\{\frac{1-\beta}{1-\eta_{2}}\right\}^{2}}}, x=\frac{p+h_{2} \sqrt{1-\left\{\frac{1-\beta}{1-\eta_{1}}\right\}^{2}}}{q-k_{2} \sqrt{1-\left\{\frac{1-\beta}{1-\eta_{2}}\right\}^{2}}}
\end{aligned}
$$

Next, express $\alpha$ in terms of $x$ by considering $w_{1}=\min \left(w_{1}, w_{2}\right), \alpha \geq 0, \alpha \leq w_{1}$ and $\alpha \leq w_{1}, \alpha \geq 0$ in eq. (7.7) we have 


$$
\alpha=w_{1} \sqrt{1-\left\{\frac{p-x q}{h_{1}+x k_{1}}\right\}^{2}}, \quad \alpha=w_{1} \sqrt{1-\left\{\frac{p-x q}{h_{1}+x k_{1}}\right\}^{2}}
$$

We get the domain of $x$

$$
x \in\left[\frac{p-h_{1}}{q+k_{1}}, \frac{p}{q}\right], \quad x \in\left[\frac{p}{q}, \frac{p+h_{1}}{q-k_{1}}\right]
$$

In the same fashion, $\beta$ can be expressed in terms of $x$ by taking $\eta_{1}=\max \left(\eta_{1}, \eta_{2}\right)$, $\beta \leq \eta_{1}, \beta \geq 0$ and $\beta \geq 0, \beta \leq \eta_{1}$ in eq. (7.8) we have

$$
\beta=1-\left(1-\eta_{1}\right) \sqrt{1-\left\{\frac{p-x q}{h_{2}+x k_{2}}\right\}^{2}}, \quad \beta=1-\left(1-\eta_{1}\right) \sqrt{1-\left\{\frac{p-x q}{h_{2}+x k_{2}}\right\}^{2}}
$$

We get the domain of $x$

$$
x \in\left[\frac{p-h_{2}}{q+k_{2}}, \frac{p}{q}\right], \quad x \in\left[\frac{p}{q}, \frac{p+h_{2}}{q-k_{2}}\right]
$$

Thus, the MF $\mu_{\frac{P}{Q}}(x)$ and NMF $v_{\frac{P}{Q}}(x)$ of $\frac{p}{Q}$ are

$$
\begin{gathered}
\mu_{\frac{P}{Q}}(x)=w_{1} \sqrt{1-\left\{\frac{p-x q}{h_{1}+x k_{1}}\right\}^{2}}, \quad \frac{p-h_{1}}{q+k_{1}} \leq x \leq \frac{p+h_{1}}{q-k_{1}} \\
\nu_{\frac{P}{Q}}(x)=1-\left(1-\eta_{1}\right) \sqrt{1-\left\{\frac{p-x q}{h_{2}+x k_{2}}\right\}^{2}}, \quad \frac{p-h_{2}}{q+k_{2}} \leq x \leq \frac{p+h_{2}}{q-k_{2}}
\end{gathered}
$$

It should be noted that fundamental operations on GSEIFNs produces GSEIFN.

\subsubsection{Illustrative examples}

Here, numerical examples of GSEIFNs are being discussed with the help of above arithmetic operations.

Suppose that $P=S_{E}(10,4,5 ; 0.2,0.5)$ and $Q=S_{E}(8,2,3 ; 0.5,0.5)$ are GSEIFNs and their respective MFs $\mu_{P}(x), \mu_{Q}(x)$ and NMFs $v_{P}(x), v_{Q}(x)$ are as follows:

$$
\begin{gathered}
\mu_{P}(x)=0.2 \sqrt{1-\left(\frac{x-10}{4}\right)^{2}}, x \in[6,14] \\
\nu_{P}(x)=1-(1-0.5) \sqrt{1-\left(\frac{x-10}{5}\right)^{2}}, x \in[5,15]
\end{gathered}
$$




$$
\begin{gathered}
\mu_{Q}(x)=0.5 \sqrt{1-\left(\frac{x-8}{2}\right)^{2}}, x \in[6,10] \\
v_{Q}(x)=1-(1-0.5) \sqrt{1-\left(\frac{x-8}{3}\right)^{2}}, x \in[5,11]
\end{gathered}
$$

The $(\alpha, \beta)$-cuts of $P$ and $Q$ are

$$
\begin{aligned}
& { }^{\alpha} P=\left[10-4 \sqrt{1-\left\{\frac{\alpha}{0.2}\right\}^{2}}, 10+4 \sqrt{1-\left\{\frac{\alpha}{0.2}\right\}^{2}}\right] \\
& { }^{\beta} P=\left[10-5 \sqrt{1-\left\{\frac{1-\beta}{1-0.5}\right\}^{2}}, 10+5 \sqrt{1-\left\{\frac{1-\beta}{1-0.5}\right\}^{2}}\right] \\
& { }^{\alpha} Q=\left[8-2 \sqrt{1-\left\{\frac{\alpha}{0.5}\right\}^{2}}, 8+2 \sqrt{1-\left\{\frac{\alpha}{0.5}\right\}^{2}}\right] \\
& { }^{\beta} Q=\left[8-3 \sqrt{1-\left\{\frac{1-\beta}{1-0.5}\right\}^{2}}, 8+3 \sqrt{1-\left\{\frac{1-\beta}{1-0.5}\right\}^{2}}\right]
\end{aligned}
$$

\subsubsection{Sum of GSEIFNs}

Sum of GSEIFNs $P$ and $Q$ is given by $P+Q$ whose MF $\mu_{P+Q}(x)$ and NMF $v_{P+Q}(x)$ are

$$
\begin{gathered}
\mu_{P+Q}(x)=0.2 \sqrt{1-\left(\frac{x-18}{6}\right)^{2}}, x \in[12,24] \\
\nu_{P+Q}(x)=1-0.5 \sqrt{1-\left(\frac{x-18}{8}\right)^{2}}, x \in[10,26]
\end{gathered}
$$

which shows this is again a GSEIFN.

\subsubsection{Subtraction of GSEIFNs}

Subtraction of GSEIFNs $P$ and $Q$ is given by $P-Q$ where MF $\mu_{P-Q}(x)$ and NMF $\nu_{P-Q}(x)$ are 


$$
\begin{gathered}
\mu_{P-Q}(x)=0.2 \sqrt{1-\left(\frac{x-2}{6}\right)^{2}}, x \in[-4,8] \\
v_{P-Q}(x)=1-0.5 \sqrt{1-\left(\frac{x-2}{8}\right)^{2}}, x \in[-6,10]
\end{gathered}
$$

which shows that subtraction of two GSEIFNs $P$ and $Q$ is again a GSEIFN.

\subsubsection{Product of GSEIFNs}

Product of GSEIFNs $P$ and $Q$ is given by $P Q$ where MF $\mu_{P Q}(x)$ and NMF $\nu_{P Q}(x)$ are

$$
\mu_{P Q}(x)=0.2 \sqrt{1-\left\{\frac{52-\sqrt{(52)^{2}-32(80-x)}}{16}\right\}^{2}},
$$

$36 \leq x \leq 140$

$$
v_{P Q}(x)=1-0.5 \sqrt{1-\left\{\frac{70-\sqrt{(70)^{2}-60(80-x)}}{30}\right\}^{2}},
$$

$25 \leq x \leq 165$

which shows that multiplication of two GSEIFNs $P$ and $Q$ is again a GSEIFN.

\subsubsection{Division of GSEIFNs}

Division of GSEIFNs $P$ and $Q$ is given by $\frac{P}{Q}$ whose MF $\mu_{\frac{P}{Q}}(x)$ and NMF $v_{\frac{P}{Q}}(x)$ are

$$
\begin{gathered}
\mu_{\frac{P}{Q}}(x)=0.2 \sqrt{1-\left(\frac{10-8 x}{4+2 x}\right)^{2}}, \quad \frac{6}{10} \leq x \leq \frac{14}{6} \\
\nu_{\frac{P}{Q}}(x)=1-0.5 \sqrt{1-\left(\frac{10-8 x}{5+3 x}\right)^{2}}, \quad \frac{5}{11} \leq x \leq \frac{15}{5}
\end{gathered}
$$

which shows that division of two GSEIFNs $P$ and $Q$ is again a GSEIFN. 


\subsection{Mean and value of GSEIFN}

Here, an attempt has been made to study mean and value of the newly defined GSEIFN by taking $(\alpha, \beta)$-cuts. Suppose that $P=S_{E}\left(p, h_{1}, h_{2} ; w_{1}, \eta_{1}\right)$ is a GSEIFN and its MF as well as NMF are as presented in Section 7.2. The mean of GSEIFN is evaluated from Definition 7.7:

$$
\begin{aligned}
M_{\mu}(P) & =\frac{1}{2} \int_{0}^{w_{1}}\left[L_{P}(\alpha)+R_{P}(\alpha)\right] d \alpha \\
& =\frac{1}{2} \int_{0}^{w_{1}}\left[p-h_{1} \sqrt{1-\left\{\frac{\alpha}{w_{1}}\right\}^{2}}+p+h_{1} \sqrt{1-\left\{\frac{\alpha}{w_{1}}\right\}^{2}}\right] d \alpha \\
& =p w_{1}
\end{aligned}
$$

Similarly for NMF $M_{v}(P)=p\left(1-\eta_{1}\right)$.

The value of GSEIFN $P$ is calculated as

$$
\begin{aligned}
V_{\mu}(P) & =\int_{0}^{w_{1}}\left[L_{P}(\alpha)+R_{P}(\alpha)\right] \alpha d \alpha \\
& =p w_{1}^{2}
\end{aligned}
$$

Similarly for NMF $V_{v}(P)=p\left(1-\eta_{1}\right)^{2}$.

\subsection{Comparing the proposed IFN with the other existing IFNs}

In this section, we compare the introduced IFN with the other existing IFNs by using some numerical examples and existing ranking approaches. Mainly we compare GSEIFN with the normal trapezoidal (NTrIFN), normal triangular IFN (NTIFN), and NSEIFN with the help Rezvani [17] and Z. Xu's [25] ranking method as discussed below.

\subsubsection{Ranking approach of Rezvani}

Rezvani [17] proposed the ranking approach for TrIFNs based on the value. We can easily evaluate the rank of two different TrIFNs by using this method. By this approach, values for the TrIFN $P=\left\langle a_{1}, a_{2}, a_{3}, a_{4} ; a_{1}{ }^{\prime}, a_{2}, a_{3}, a_{4}^{\prime}\right\rangle$ are evaluated as 


$$
V_{\mu}(A)=\frac{a_{1}+2 a_{2}+2 a_{3}+a_{4}}{6}
$$

For two different TrIFNs $A$ and $B$, rank can be evaluated by using the following procedure:

$$
\begin{aligned}
& \text { If } V_{\mu}(A)>V_{\mu}(B) \text { then } A>B \\
& \text { If } V_{\mu}(A) \leq V_{\mu}(B) \text { then } A \leq B
\end{aligned}
$$

This approach gives the counterintuitive output in some situations. For example, for the NTrIFNs $A=\langle(4,5.5,6,8) ; 1,0\rangle$ and $B=\langle(3.5,5,7,7.5) ; 1,0\rangle$ values of $A$ and $B$ denoted by $V_{\mu} A$ and $V_{\mu} B$ are evaluated as

$$
\begin{aligned}
& V_{\mu}(A)=\frac{4+2 \times 5.5+2 \times 6+8}{6}=5.833 \\
& V_{\mu}(B)=\frac{3.5+2 \times 5+2 \times 7+7.5}{6}=5.833
\end{aligned}
$$

This gives the same output for non identical NTrIFNs $A$ and $B$. It is problematic for the decision makers to identify the best one. To get rid of this situation, NTrIFN can be reconstructed in the form of GSEIFN as given below.

Suppose that $A=S_{E}(6,2,2 ; 0.5,0.5)$ and $B=S_{E}(5.5,2,2 ; 0.1,0.7)$ are two GSEIFNs whose MFs and NMFs are

$$
\begin{gathered}
\mu_{(A)(x)}=0.5 \sqrt{1-\left\{\frac{x-6}{2}\right\}^{2}}, \quad x \in[4,6] \\
v_{(B)(x)}=1-0.5 \sqrt{1-\left\{\frac{x-6}{2}\right\}^{2}}, \quad x \in[4,6]
\end{gathered}
$$

and

$$
\begin{gathered}
\mu_{(B)(x)}=0.1 \sqrt{1-\left\{\frac{x-5.5}{2}\right\}^{2}}, \quad x \in[3.5,7.5] \\
\nu_{(B)(x)}=1-0.3 \sqrt{1-\left\{\frac{x-5.5}{2}\right\}^{2}}, \quad x \in[3.5,7.5]
\end{gathered}
$$

Then $\alpha$-cuts and $\beta$-cuts of $A$ and $B$ are

$$
{ }^{\alpha} A=\left[6-2 \sqrt{1-\left\{\frac{\alpha}{0.5}\right\}^{2}}, 6+2 \sqrt{1-\left\{\frac{\alpha}{0.5}\right\}^{2}}\right]
$$




$$
\begin{gathered}
{ }^{\alpha} B=\left[5.5-2 \sqrt{1-\left\{\frac{\alpha}{0.1}\right\}^{2}}, 5.5+2 \sqrt{1-\left\{\frac{\alpha}{0.1}\right\}^{2}}\right] \\
{ }^{\beta} A=\left[6-2 \sqrt{1-\left\{\frac{1-\beta}{0.5}\right\}^{2}}, 6+2 \sqrt{1-\left\{\frac{1-\beta}{0.5}\right\}^{2}}\right] \\
{ }^{\beta} B=\left[5.5-2 \sqrt{1-\left\{\frac{1-\beta}{0.3}\right\}^{2}}, 5.5+2 \sqrt{1-\left\{\frac{1-\beta}{0.3}\right\}^{2}}\right]
\end{gathered}
$$

Through Definition 7.5, the values for $A$ and $B$ can be measured as

$$
\begin{gathered}
V_{\mu}(A)=\int_{0}^{0.5}\left(6-2 \sqrt{1-\left\{\frac{\alpha}{0.5}\right\}^{2}}+6+2 \sqrt{1-\left\{\frac{\alpha}{0.5}\right\}^{2}}\right) \alpha d \alpha=1.5 \\
V_{\mu}(B)=\int_{0}^{0.1}\left(5.5-2 \sqrt{1-\left\{\frac{\alpha}{0.1}\right\}^{2}}+5.5+2 \sqrt{1-\left\{\frac{\alpha}{0.1}\right\}^{2}}\right) \alpha d \alpha=0.055
\end{gathered}
$$

which shows that $V_{\mu}(A)>V_{\mu}(B)$ and this indicates that $A>B$. It is witness that drawbacks of earlier method can be overcome.

\subsubsection{Ranking approach of Xu}

Using score and accuracy function, $\mathrm{Xu}$ [25] developed this ranking approach [25]. Suppose that $A=\left\langle a_{1}, a_{2}, a_{3} ; w_{1}, \eta_{1}\right\rangle$ and $B=\left\langle b_{1}, b_{2}, b_{3} ; w_{2}, \eta_{2}\right\rangle$ are two triangular IFNs. Then by Xu's ranking method, score functions denoted by $S(A), S(B)$ and accuracy functions denoted by $H(A), H(B)$ are as follows:

$$
S(A)=w_{1}-\eta_{1}, S(B)=w_{2}-\eta_{2}, H(A)=w_{1}+\eta_{1}, H(B)=w_{2}+\eta_{2}
$$

Then the rank of $A$ and $B$ can be determined by using the following procedure:

If $S(A)<S(B)$ then $A<B$

If $S(A)=S(B)$ then we check the value of accuracy function

If $H(A)>H(B)$ then $A>B$

If $H(A) \leq H(B)$ then $A \leq B$

For the two NTIFNs $A=\langle(4,5.5,6) ; 1,0\rangle$ and $B=\langle(3.5,5,7) ; 1,0\rangle$, using Xu's approach the score and accuracy values are obtained as

$$
S(A)=1, S(B)=1, H(A)=1, H(B)=1
$$

which provides the identical result. 
Similarly, NSEIFNs $A=\langle(4,2,3) ; 1,0\rangle$ and $B=\langle(8,3,5) ; 1,0\rangle$ whose MFs and NMFs are

$$
\begin{gathered}
\mu_{(A)(x)}=\sqrt{1-\left\{\frac{x-4}{2}\right\}^{2}}, x \in[2,6] \\
\nu_{(B)(x)}=1-\sqrt{1-\left\{\frac{x-4}{3}\right\}^{2}}, x \in[1,7]
\end{gathered}
$$

and

$$
\begin{gathered}
\mu_{(B)(x)}=\sqrt{1-\left\{\frac{x-8}{3}\right\}^{2}}, x \in[5,11] \\
v_{(B)(x)}=1-\sqrt{1-\left\{\frac{x-8}{5}\right\}^{2}}, x \in[3,13]
\end{gathered}
$$

For these IFNs, value score and accuracy functions are

$$
S(A)=1, S(B)=1, H(A)=1, H(B)=1
$$

These IFNs also provide identical results. So it is difficult for the experts to distinguish the best alternative by using NSEIFN. So we reconstruct the IFN in the form of GSE type. Suppose that $A=S_{E}(4,2,3 ; 0.1,0.05)$ and $B=S_{E}(8,3,5 ; 0.2,0.03)$ whose MF and NMFs are

$$
\begin{gathered}
\mu_{(A)(x)}=0.1 \sqrt{1-\left\{\frac{x-4}{2}\right\}^{2}}, x \in[2,6] \\
\nu_{(B)(x)}=1-0.95 \sqrt{1-\left\{\frac{x-6}{3}\right\}^{2}}, x \in[3,9]
\end{gathered}
$$

and

$$
\begin{gathered}
\mu_{(B)(x)}=0.2 \sqrt{1-\left\{\frac{x-8}{3}\right\}^{2}}, x \in[5,11] \\
v_{(B)(x)}=1-0.97 \sqrt{1-\left\{\frac{x-8}{5}\right\}^{2}}, x \in[3,13]
\end{gathered}
$$

Then the values of score functions and accuracy functions are

$$
S(A)=0.05, S(B)=0.17, H(A)=0.15, H(B)=0.23
$$

which shows that $A<B$. From this comparative analysis, it can be opined that the present approach has the ability to overcome those drawbacks of NTIFNs and NSEIFNs. 


\subsection{Ranking approach of IFN based on consummated exponential mean}

Here, a novel ranking approach of IFNs based on the consummated exponential mean has been presented.

Let $B$ be an IFN whose $(\alpha, \beta)$-cuts are considered as follows:

$$
{ }^{\alpha} B=\left[L_{B}(\alpha), R_{B}(\alpha)\right],{ }^{\beta} B=\left[L_{B}(\beta), R_{B}(\beta)\right]
$$

Now, evaluate the mean of $B$ as

$$
\begin{aligned}
& M_{\mu}(B)=\frac{1}{2} \int_{0}^{w}\left[L_{B}(\alpha)+R_{B}(\alpha)\right] d \alpha \\
& M_{\nu}(B)=\frac{1}{2} \int_{\eta}^{1}\left[L_{B}(\beta)+R_{B}(\beta)\right] d \beta
\end{aligned}
$$

for normal $w=1$ and $\eta=0$.

Now the consummated exponential mean of $B$ is given by $M(B)$ and is defined as

$$
M(B)=e^{M_{\mu}(B)}+e^{M_{\nu}(B)}
$$

Suppose that $P$ and $Q$ are two IFNs, then the ranking procedure is as follows:

$$
\begin{aligned}
& \text { If } M(P)>M(Q) \text { then } P>Q \\
& \text { If } M(P) \leq M(Q) \text { then } P \leq Q
\end{aligned}
$$

Consummated exponential mean of generalized trapezoidal IFN (GTrIFN) $P=<\left[p_{1}, p_{2}, p_{3}, p_{4} ; w_{1}\right],\left[p_{1}^{\prime}, p_{2}, p_{3}, p_{4}^{\prime} ; \eta_{1}\right]>$ is defined by

$$
M(P)=e^{\left\{\frac{w_{1}}{4}\left(p_{1}+p_{2}+p_{3}+p_{4}\right)\right\}}+e^{\left\{\frac{\left(1-\eta_{1}\right)}{4}\left(p_{1}^{\prime}+p_{2}+p_{3}+p_{4}^{\prime}\right)\right\}}
$$

Consummated exponential mean of generalized triangular IFN (GTIFN) $P<$ $\left[p_{1}, p_{2}, p_{4} ; w_{1}\right],\left[p_{1}^{\prime}, p_{2}, p_{4}^{\prime} ; \eta_{1}\right]>$ is defined by

$$
M(P)=e^{\left\{\frac{w_{1}}{4}\left(p_{1}+2 p_{2}+p_{4}\right)\right\}}+e^{\left\{\frac{\left(1-\eta_{1}\right)}{4}\left(p_{1}^{\prime}+2 p_{2}+p_{4}^{\prime}\right)\right\}}
$$

Consummated exponential mean of GSEIFN $P=\left(p, h_{1}, h_{2} ; w_{1}, \eta_{1}\right)$ is defined by

$$
M(P)=e^{p w_{1}}+e^{p\left(1-\eta_{1}\right)}
$$

\subsubsection{Numerical illustration}

Here, a discussion has been made on some numerical examples with the help of proposed ranking approach. Suppose that $P=\langle(1,2,3,4 ; 0.2),(0.5,2,3,4.5 ; 0.4)\rangle$ 
are two GTrIFNs. Then the consummated exponential mean of $P$ and $Q$ are evaluated as follows:

$$
\begin{aligned}
M(P) & =e^{\left\{\frac{0.2}{4}(1+2+3+4)\right\}}+e^{\left\{\frac{(1-0.4)}{4}(0.5+2+3+4)\right\}} \\
& =e^{0.5}+e^{1.5} \\
& =6.130411
\end{aligned}
$$

Similarly, $M(Q)=3.799609$, which shows that $M(P)>M(Q)$, that is the rank of order $P>Q$.

Suppose that $P=\langle(1,2,3 ; 0.2),(0.5,2,3 ; 0.4)\rangle$ are two GTIFNs. Then the consummated exponential mean of $P$ and $Q$ are evaluated as follows:

$$
\begin{aligned}
M(P) & =e^{\left\{\frac{0.2}{4}(1+2+3)\right\}}+e^{\left\{\frac{(1-0.4)}{4}(0.5+2+3)\right\}} \\
& =e^{0.4}+e^{1.125} \\
& =4.572042
\end{aligned}
$$

Similarly, $M(Q)=2.645911$,

which shows that $M(P)>M(Q)$, that is, the rank of order $P>Q$.

Suppose that $P=S_{E}(5,2,3 ; 0.2,0.3)$ and $P=S_{E}(4,1,2 ; 0.1,0.3)$ are two GSEIFNs, then the consummated exponential mean of $P$ and $Q$ are

$$
M(P)=4.137349, \quad M(Q)=3.717366
$$

which shows that $M(P)>M(Q)$, that is, the rank of order $P>Q$.

\subsubsection{Comparative study of the present approach with the available approaches}

Here, we compare the present ranking approach with the other existing methods in literature as shown in Table 7.1.

From the above table it is observed that the ranking approaches developed in $[6,10,15,17,26,27]$ as well as in [9] produce the same result for nonidentical IFNs. To overcome these drawbacks and to get a proper result, we should develop a proper ranking approach. In this chapter, we introduced a consummated exponential meanbased ranking approach and from the comparative Table 7.1, it is encountered that the present ranking approach has the capability to overcome the limitations of the available methods. 
Table 7.1: Comparative table.

\begin{tabular}{|c|c|c|}
\hline IFNs & $\begin{array}{l}\text { Available } \\
\text { approaches }\end{array}$ & $\begin{array}{l}\text { Present } \\
\text { approach }\end{array}$ \\
\hline$P=\langle(4,5.5,6) ; 1,0\rangle, Q=\langle(3.5,5,7) ; 1,0\rangle$ & {$[25], P=Q$} & $P>Q$ \\
\hline$P=\langle(0.45,0.63,0.83) ; 0.73,0.2\rangle, Q=\langle(0.48,0.64,0.82) ; 0.72,0.2\rangle$ & {$[10], P=Q$} & $P<Q$ \\
\hline$P=\langle(0,0.25,0.3) ; 1,0\rangle, Q=\langle(0.1,0.2,0.4) ; 1,0\rangle$ & {$[15], P=Q$} & $P<Q$ \\
\hline$P=\langle(0,0.25,0.3) ; 1,0\rangle, Q=\langle(0.1,0.2,0.4) ; 1,0\rangle$ & {$[6], P=Q$} & $P<Q$ \\
\hline$P=\langle(0.20,0.35,0.40,0.55) ; 1,0\rangle, Q=\langle(0.10,0.40,0.45,0.45) ; 1,0\rangle$ & {$[26], P=Q$} & $P>Q$ \\
\hline$P=\langle(4,5.5,6,8) ; 1,0\rangle, Q=\langle(3.5,5,7,7.5) ; 1,0\rangle$ & {$[17], P=Q$} & $P>Q$ \\
\hline$P=\langle(2.57,2.73,2.83) ; 0.73,0.2\rangle, Q=\langle(2.58,2.74,2.82) ; 0.72,0.2\rangle$ & {$[27], P=Q$} & $P>Q$ \\
\hline$P=\langle(3.5,5,7,7.5) ; 0.5,0.5\rangle, Q=\langle(4,5.5,6,8) ; 0.7,0.3\rangle$ & [7], $P>Q$ & $P<Q$ \\
\hline$P=\langle(0.20,0.35,0.40,0.55) ; 1,0\rangle, Q=\langle(0.10,0.40,0.45,0.45) ; 1,0\rangle$ & {$[28], P<Q$} & $P>Q$ \\
\hline$P=\langle(4,4,4) ; 1,0\rangle, Q=\langle(2,2,2) ; 1,0\rangle$ & [9], $P=Q$ & $P>Q$ \\
\hline
\end{tabular}

\subsection{MCDM via GSEIFN using the present ranking approach}

Here, a methodology of MCDM problem based on the proposed ranking method and proposed IFN has been discussed.

Suppose that $P=\left\{P_{1}, P_{2}, \ldots, P_{n}\right\}$ be a set of $m$ alternatives and $N=\left\{N_{1}, N_{2}, \ldots, N_{m}\right\}$ be a set of $n$ criteria for each alternative $P_{i}$. Let $w_{c}=\left(w_{c_{1}}, w_{c_{2}}, \ldots, w_{c_{m}}\right)$ be the weight of criteria where $w_{c_{n}} \in[0,1]$ and $w_{c_{1}}+w_{c_{2}}+\cdots+w_{c_{n}}=1$ to choose the best alternative among $P_{i}{ }^{\prime} s$ for $i=1,2, \ldots ., n$ based on the consummated exponential mean method. The methodology is discussed as follows:

Step 1: Form a table of fuzzy rating criteria by choosing fuzzy criteria in terms of GSEIFNs $p_{i j}=\left(p_{i j} ; w_{i j}, \eta_{i j}\right),(i=1,2,3, \ldots, m ; j=1,2,3, \ldots, n)$ and $p_{i j} \in R$ and weight of criteria $w_{c_{n}}$.

Step 2: A decision matrix $B=\left(p_{i j}\right)_{m \times n}$ has been formed by using the above choosing table of fuzzy rating criteria:

$$
B=\begin{gathered}
N_{1} \\
N_{2} \\
\vdots \\
N_{n}
\end{gathered}\left[\begin{array}{cccc}
P_{1} & P_{2} & \ldots & P_{n} \\
\left(p_{11} ; w_{11}, \eta_{11}\right) & \left(p_{12} ; w_{12}, \eta_{12}\right) & \ldots & \left(p_{1 n}, ; w_{1 n}, \eta_{1 n}\right) \\
\left(p_{21} ; w_{21}, \eta_{21}\right) & \left(p_{22} ; w_{22}, \eta_{22}\right) & \ldots & \left(p_{2 n} ; w_{2 n}, \eta_{2 n}\right) \\
\ldots & \ldots & \ldots & \ldots \\
\left(p_{m 1} ; w_{m 1}, \eta_{m 1}\right) & \left(b_{m 1} ; w_{m 2}, \eta_{m 2}\right) & \ldots & \left(b_{m n} ; w_{m n}, \eta_{m n}\right)
\end{array}\right]
$$


Step 3: If the given data are inadequate, then normalize the choosing decision matrix $B=\left(b_{i j}\right)_{m \times n}$ by using the equation

$$
a_{i j}=\frac{w_{c_{i}} p_{i j}}{100}
$$

Then the normalized decision matrix will be of the form

$$
R=\begin{gathered}
N_{1} \\
N_{2} \\
\vdots \\
N_{m}
\end{gathered}\left[\begin{array}{cccc}
P_{1} & P_{2} & \ldots & P_{n} \\
\left(a_{11} ; w_{11}^{\prime}, \eta_{11}^{\prime}\right) & \left(a_{12} ; w_{12}^{\prime}, \eta_{12}^{\prime}\right) & \ldots & \left(a_{1 n} ; w_{1 n}^{\prime}, \eta_{1 n}^{\prime}\right) \\
\left(a_{21} ; w_{21}^{\prime}, \eta_{21}^{\prime}\right) & \left(a_{22} ; w_{22}^{\prime}, \eta_{22}^{\prime}\right) & \ldots & \left(a_{2 n} ; w_{2 n}^{\prime}, \eta_{2 n}^{\prime}\right) \\
\ldots & \ldots & \ldots & \ldots \\
\left(a_{m 1} ; w_{m 1}^{\prime}, \eta_{m 1}^{\prime}\right) & \left(a_{m 1} ; w_{m 2}^{\prime}, \eta_{m 2}^{\prime}\right) & \ldots & \left(a_{m n} ; w_{m n}^{\prime}, \eta_{m n}^{\prime}\right)
\end{array}\right]
$$

Step 4: After getting the normalized decision matrix $R=\left(a_{i j}\right)_{m \times n}$, evaluate $R\left(P_{i}\right)$ by using the following equation:

$$
R\left(P_{i}\right)=\sum_{k=1}^{m} M\left(P_{i}\right)_{N_{k}}, i=1,2, \ldots, n
$$

where $M\left(P_{i}\right)_{N_{k}}$ is the consummated exponential mean-based rank of GPIFNs.

Step 5: The decision makers choose the alternative of maximum $R\left(P_{i}\right)$.

\subsubsection{Numerical example of MCDM}

In this section, an MCDM problem has been discussed to exhibit the validity and applicability of the present ranking method and proposed IFN. Suppose that a student searching for a good college among available colleges, namely, $P_{1}, P_{2}, P_{3}$ for him located in a city on the basis of four criteria represented by GSEIFNs:

(1) $N_{1}=$ Transportation

(2) $N_{2}=$ Yearly cost

(3) $N_{3}=$ Teaching quality

(4) $N_{4}=$ Yearly performance

Let the weight of criteria $w_{c}=(0.1,0.2,0.3,0.4)$ and the fuzzy rating of criteria of each alternative as given in Table 7.2. 
The decision matrix $B=\left(b_{i j}\right)$ is formed by using Table 7.2:

Table 7.2: Rating of criteria.

\begin{tabular}{llll}
\hline & $\boldsymbol{P}_{\mathbf{1}}$ & $\boldsymbol{P}_{\mathbf{2}}$ & $\boldsymbol{P}_{\mathbf{3}}$ \\
\hline$N_{1}$ & $(40,20,30 ; 0.2,0.3)$ & $(90,40,50 ; 0.4,0.5)$ & $(50,30,40 ; 0.3,0.4)$ \\
\hline$N_{2}$ & $(30,10,20 ; 0.1,0.2)$ & $(40,20,30 ; 0.2,0.3)$ & $(60,40,50 ; 0.2,0.5)$ \\
\hline$N_{3}$ & $(50,30,40 ; 0.3,0.4)$ & $(80,30,40 ; 0.4,0.5)$ & $(90,40,50 ; 0.4,0.5)$ \\
\hline$N_{4}$ & $(60,40,50 ; 0.2,0.5)$ & $(70,20,50 ; 0.3,0.5)$ & $(90,40,50 ; 0.4,0.5)$ \\
\hline
\end{tabular}

Table 7.3: Table of ranking result by the proposed method.

\begin{tabular}{lr}
\hline $\begin{array}{l}\text { Alternatives of } \\
\text { decision-making problem }\end{array}$ & $\begin{array}{r}\text { Results by using } \\
\text { proposed method }\end{array}$ \\
\hline$P_{1}$ & 8.5352206 \\
\hline$P_{2}$ & 8.7712329 \\
\hline$P_{3}$ & 8.9213067 \\
\hline
\end{tabular}

$$
B=\left[\begin{array}{ccc}
(40,20,30 ; 0.2,0.3) & (90,40,50 ; 0.4,0.5) & (50,30,40 ; 0.3,0.4) \\
(30,10,20 ; 0.1,0.2) & (40,20,30 ; 0.2,0.3) & (60,40,50 ; 0.2,0.5) \\
(50,30,40 ; 0.3,0.4) & (80,30,40 ; 0.4,0.5) & (90,40,50 ; 0.4,0.5) \\
(60,40,50 ; 0.2,0.5) & (70,20,50 ; 0.3,0.5) & (90,40,50 ; 0.4,0.5)
\end{array}\right]
$$

Now normalized the above matrix by using eq. (7.9) as follows:

$$
\begin{aligned}
R= & \left.\begin{array}{ll}
(0.04,0.02,0.03 ; 0.0002,0.0003) & (0.09,0.04,0.05 ; 0.0004,0.0005) \\
(0.06,0.02,0.04 ; 0.0002,0.0004) & (0.08,0.04,0.06 ; 0.0004,0.0006) \\
(0.15,0.09,0.12 ; 0.0009,0.0012) & (0.24,0.09,0.12 ; 0.0012,0.0015) \\
(0.24,0.16,0.2 ; 0.0008,0.002) & (0.28,0.08,0.2 ; 0.0012,0.002) \\
& (0.05,0.03,0.04 ; 0.0003,0.0004) \\
& (0.12,0.08,0.1 ; 0.004,0.001) \\
& (0.27,0.12,0.15 ; 0.0012,0.0015) \\
& (0.36,0.16,0.2 ; 0.0016,0.002)
\end{array}\right]
\end{aligned}
$$

By using eq. (7.10), result of the decision-making problem is obtained.

By observing Table 7.3 we have $P_{3}>P_{2}>P_{1}$; therefore, $P_{3}$ college will be chosen by the student. 


\subsubsection{Conclusion}

GSEIFN has been introduced in this chapter. Here we performed some arithmetic operations via $(\alpha, \beta)$-cut technique and it is exhibited that fundamental operations on GSEIFNs also give GSEIFN. There are several types of IFNs that have been developed by many researchers. In most of the MCDM problems, researchers used the data or information that are in terms of trapezoidal and triangular IFNs. But in some cases, these IFNs give incorrect solutions by using NTrIFN, NTIFN, and NSEIFN. In this chapter, we have shown that the proposed IFN overcomes these limitations in NTrIFN, NTIFN, and NSEIFN, although a new ranking method has been developed by using the exponential mean of MF and NMF and comparing this method with the other existing methods with the help of some existing ranking approaches and numerical examples. Finally, an MCDM problem has been done by using the proposed IFN and the present novel ranking method to showcase the validity and applicability.

\section{References}

[1] Zadeh L.A. (1965) Fuzzy sets, Information and Control, 8(3), 338-353.

[2] Atanassov K.T. Intuitionistic fuzzy sets, In: Intuitionistic fuzzy sets, Physica, Heidelberg, 1999, 1-137.

[3] Prakash K.A., Suresh M., \& Vengataasalam S. (2016) A new approach for ranking of intuitionistic fuzzy numbers using a centroid concept, Mathematical Sciences, 10(4), 177-184.

[4] Sudha A.S. \& Vijayalakshmi K. (2017) A value and ambiguity-based ranking of symmetric hexagonal intuitionistic fuzzy numbers in decision making, Advanced Fuzzy Mathematics, 12(4), 867-879.

[5] Dutta P. \& Saikia B. (2019) Arithmetic operations on normal semielliptic intuitionistic fuzzy numbers and their application in decision-making, Granular Computing, 1-17.

[6] Li D.F., Nan J.X., \& Zhang M.J. (2010) A ranking method of triangular intuitionistic fuzzy numbers and application to decision making, International Journal of Computational Intelligence Systems, 3(5), 522-530.

[7] Das S. \& Guha D. (2013) Ranking of intuitionistic fuzzy number by centroid point, Journal of Industrial and Intelligent Information, $1,2$.

[8] Roseline S. \& Amirtharaj H. (2015) Methods to find the solution for the intuitionistic fuzzy assignment problem with ranking of intuitionistic fuzzy numbers, International Journal of Innovative Research in Science, Engineering and Technology, 4, 7.

[9] Darehmiraki M. (2019) A novel parametric ranking method for intuitionistic fuzzy numbers, Iranian Journal of Fuzzy Systems, 16, 1.

[10] Jianqiang W. \& Zhong Z. (2009) Aggregation operators on intuitionistic trapezoidal fuzzy number and its application to multi-criteria decision making problems, Journal of Systems Engineering and Electronics, 20(2), 321-326.

[11] Velu L.G.N., Selvaraj J., \& Ponnialagan D. (2017) A new ranking principle for ordering trapezoidal intuitionistic fuzzy numbers, Complexity. 
[12] Rezvani S. (2015) Ranking generalized exponential trapezoidal fuzzy numbers based on variance, Applied Mathematics and Computation, 262, 191-198.

[13] Garg H. \& Kumar K. (2019) Improved possibility degree method for ranking intuitionistic fuzzy numbers and their application in multiattribute decision-making, Granular Computing, 4(2), 237247.

[14] Nehi H.M. (2010) A new ranking method for intuitionistic fuzzy numbers, International Journal of Fuzzy Systems, 12(1), 8086.

[15] Li D.F. (2010) A ratio ranking method of triangular intuitionistic fuzzy numbers and its application to madm problems, Computers \& Mathematics with Applications, 60(6), 1557-1570.

[16] Dubey D. \& Mehra A. Linear programming with triangular intuitionistic fuzzy number, 2011, 563-569.

[17] Rezvani S. (2013) Ranking method of trapezoidal intuitionistic fuzzy numbers, Annals of Fuzzy Mathematics and Informatics, 5(3), 515-523.

[18] Zeng X., Li D., \& Yu G. 2014 A value and ambiguity-based ranking method of trapezoidal intuitionistic fuzzy numbers and application to decision making, The Scientific World Journal.

[19] Li D.F. \& Yang J. (2015) A difference-index based ranking method of trapezoidal intuitionistic fuzzy numbers and application to multiattribute decision making, Mathematical and Computational Applications, 20(1), 25-38.

[20] Das D. \& De P.K. (2015) On ranking of trapezoidal intuitionistic fuzzy numbers and its application to multi attribute group decision making, Journal of New Theory(6), 99-108.

[21] Bharati S. (2017) Ranking method of intuitionistic fuzzynumbers, Global Journal of Pure and Applied Mathematics, 13(9), 4595-4608.

[22] Beaula T. \& Priyadharsini M. (2015) Fuzzy transportation problem with the value and ambiguity indices of trapezoidal intuitionistic fuzzy numbers, Malaya Journal, Matematik S, (2), 427437.

[23] Jamkhaneh E.B. (2016) A value and ambiguity-based ranking method of generalized intuitionistic fuzzy numbers, Research and Communications in Mathematics and Mathematical Sciences, 6(2), 89103.

[24] Nayagam V.L.G., Jeevaraj S., \& Dhanasekaran P. (2018). An improved ranking method for comparing trapezoidal intuitionistic fuzzy numbers and its applications to multicriteria

[25] Xu Z. (2007) Intuitionistic fuzzy aggregation operators, IEEE Transactions on Fuzzy Systems, 15(6), 1179-1187.

[26] De P.K. \& Das D. (2012). Ranking of trapezoidal intuitionistic fuzzy numbers. 12th International Conference on Intelligent Systems Design and Applications(ISDA), Kochi, India, pp.184-188

[27] Wu J. \& Cao Q. (2013) Same families of geometric aggregation operators with intuitionistic trapezoidal fuzzy numbers, Applied Mathematical Modelling, 37(1-2), 318327.

[28] Nayagam V.L.G., Jeevaraj S., \& Sivaraman G. (2016) Complete ranking of intuitionistic fuzzy numbers, Fuzzy Information and Engineering, 8(2), 237-254. 